\title{
Preface: progress in synthesis and characterization of energy materials
}

\author{
Thomas Höche • Anke Weidenkaff • \\ Ichiro Terasaki
}

Received: 10 December 2012/ Accepted: 10 December 2012/Published online: 20 December 2012

(C) Springer Science+Business Media New York 2012

The looming shortage of natural resources drives the scientific and commercial interest in renewable energies, energy recovery, and power saving. In this context, the development of novel approaches, replacement of toxic and less available resources, and improvement of existing technologies to synthesize suitable materials is pivotal. This is reflected in more than 160,000 papers found when searching for "energy materials" on the Web of Science. Knowledge-based materials engineering is mostly based upon the micro-/nanostructure-property relationships.

Staying abreast with this key challenge of the twenty first century, two symposia devoted to the progress in synthesis and characterization of materials for energy were held under the auspices of the E-MRS 2012 Spring Meeting in Strasbourg, France, May 14-18, 2012.

The first symposium, entitled "Quantitative microscopy of energy materials", was organized by Aïcha HesslerWyser, Thomas Höche, Hugo Bender, and Wolfgang Jäger. About 70 contributions, including thirteen invited talks, from 23 countries (Europe as well as the USA, Japan, Korea, China, and Israel) covered a wide range of microstructure characterization techniques (such as atomic force

T. Höche $(\bowtie)$

Center for Applied Microstructure Diagnostics (CAM), Fraunhofer Institute for Mechanics of Materials IWM,

Walter-Huelse-Strasse 1, 06120 Halle, Germany

e-mail: thomas.hoeche@iwmh.fraunhofer.de

\section{A. Weidenkaff}

Solid State Chemistry and Catalysis, Swiss Federal Laboratories for Materials Science and Technology, Überlandstrasse 129, 8600 Dübendorf, Switzerland

I. Terasaki

Department of Physics, Nagoya University, Nagoya 464-8602, Japan microscopy, transmission electron microscopy and spectroscopy, and scanning electron microscopy). The application of cutting edge analytics was shown for a huge spectrum of materials, like for H-production and storage, fuel cells, batteries, reactors, and silicon and organic photovoltaics.

In the second symposium "Unconventional Thermoelectrics: From New Materials to Energy Conversion Devices", Anke Weidenkaff, Antoine Maignan, Heiner Linke, and Ichiro Terasaki bundled contributions from the rapidly growing thermoelectrics community. The main objective of this convention was to show how advances in thermoelectric materials theory, synthesis, and characterization can accelerate the improvement of waste heat recovery devices. Thermoelectricity is a multidisciplinary field, and the symposium reflected this diversity. It attracted a scientifically diverse audience of more than 100 graduate students, post-doctoral researchers, academics, and industrial researchers. The symposium included eleven invited speakers, 60 oral presentations, and 50 posters.

In this special section of the Journal of Materials Science, peer-reviewed, full archival papers based on current research results presented at the above-mentioned symposia are pooled. The range of thermoelectrics (TE) materials reached from tellurides, skutterudites, intermetallics, complex oxides and sulfides to organic semiconductors and nanocomposite materials. Aspects of TE device design and application, state-of-the-art semiconductor technology was employed and demonstrated to enhance thermoelectric energy conversion efficiency. Complex oxide gate FET and Si-nano-layer structure are prime examples. Various techniques to evaluate thermal conductivity at the nano scale are being developed, and it is becoming clear how nanostructuring improves the thermoelectric performance. The aforementioned contributions are complemented by articles dealing with oxide formation 
on stainless steels exposed to simulated harsh environments, with growth mechanisms of functional thin films, with in situ TEM investigations of nickel oxide reduction, with the aging of organic solar cell stacks, with the microstructure of inkjetprinted organic photovoltaic structures, and with phase transformations affecting thermal expansion properties of low thermal expansion composites. Besides these experimental accounts, theoretical contributions devoted to effective transport properties of porous layers in electrolysis and fuel cells and hybrid porous nanotube crystal networks have been included.

The guest editors are indebted to all authors for their time and efforts to enable this valuable record of two enjoyable symposia!
The symposia organizers acknowledge substantial financial support of the following companies and organizations (in alphabetic order): Carl Zeiss NTS GmbH, CEOS GmbH, European Microscopy Society EMS, FEI Company, Gatan GmbH, Hitachi High-Technologies Europe GmbH, Swiss Federal Office of Energy SFOE, Swiss Thermoelectric Society STS, and Sigma Aldrich. Their generosity allowed us to invite numerous distinguished speakers setting highlights to our symposia. Last but not least, we are very grateful to our co-chairs, Aïcha Hessler-Wyser, Wolfgang Jäger, Hugo Bender, Antoine Maignan, and Heiner Linke who contributed to the success of the symposia and to the papers derived from them. 